\title{
Etomidate infusion at low doses is an effective and safe treatment for severe Cushing's syndrome outside intensive care
}

\section{Stefan M Constantinescu ${ }^{1}$, Natacha Driessens ${ }^{2}$, Aurélie Lefebvre ${ }^{3}$, Raluca M Furnica', Bernard Corvilain ${ }^{2}$ and Dominique Maiter ${ }^{1}$}

'Endocrinology Department, Cliniques Universitaires Saint Luc, Brussels, Belgium, ${ }^{2}$ Endocrinology Department, Cliniques Universitaires de Bruxelles Hôpital Erasme, Bruxelles, Belgium, and ${ }^{3}$ Endocrinology Department, Cliniques Saint Pierre, Ottignies, Belgium
Correspondence should be addressed to D Maiter Email dominique.maiter@ uclouvain.be

\begin{abstract}
Introduction: Intravenous etomidate infusion is effective to rapidly lower cortisol levels in severe Cushing's syndrome (CS) in the intensive care unit (ICU). Recently, etomidate treatment has also been proposed at lower doses in non-ICU wards, but it is not yet clear how this approach compares to ICU treatment.

Methods: We compared data from patients with severe CS treated with high starting doses of etomidate (median: $0.30 \mathrm{mg} / \mathrm{kg}$ BW/day) in ICU or with lower starting doses (median: $0.025 \mathrm{mg} / \mathrm{kg}$ BW/day) in non-ICU medical wards. Results: Fourteen patients were included, among which ten were treated with low starting doses (LD) and four with high starting doses etomidate (HD). All patients had severe and complicated CS related to adrenal carcinoma $(n=8)$ or ectopic ACTH secretion $(n=6)$. Etomidate was effective in reducing cortisol levels below $500 \mathrm{nmol} / \mathrm{L}$ in a median of 1 day in the HD group compared to 3 days in the LD group $(P=0.013)$. However, all patients of the HD group had etomidate-induced cortisol insufficiency and needed frequent monitoring, while no patient from the LD group required hydrocortisone supplementation. No patient in either group died from complications of CS or etomidate treatment, but final outcome was poor as six patients in the LD group and all four patients in the HD group died from their cancer during follow-up.

Conclusion: Our study suggests that, for patients with severe CS who do not require intensive organ-supporting therapy, the use of very low doses of etomidate in medical wards should be considered.
\end{abstract}

\section{Introduction}

Severe Cushing's syndrome (CS) is usually defined by serum cortisol levels above $1000 \mathrm{nM}$ or urinary free cortisol (UFC) five times above the upper limit of normal (ULN) range in a 24-h urine collection, associated with severe and potentially life-threatening complications (1). Such severe hypercortisolism is most frequently caused by ectopic ACTH secretion (2) (EAS) or by adreno-cortical carcinoma (3) (ACC). Rapid control of cortisol levels is essential as it has been shown to decrease complications and subsequent peri-operative mortality (4).
Single or combined treatments with the two oral anticortisolic drugs, ketoconazole and metyrapone, can be effective in treating severe $\operatorname{CS}(4,5,6)$ but are not sufficiently rapid and powerful in acute situations. Moreover, severe and acute illness or surgery may render oral treatment inappropriate and metyrapone is not readily available in many countries, including Belgium.

Etomidate is an imidazole derivative widely available and used as hypnotic agent in general anesthesia induction, while also being one of the most potent

Published by Bioscientifica Ltd. 
inhibitors of $11 \beta$-hydroxylase in adrenal steroidogenesis (7). In a seminal article, Schulte et al. showed that etomidate given at the dose of $0.30 \mathrm{mg} / \mathrm{kg} / \mathrm{h}$ was effective in rapidly reducing cortisol levels in patients with CS, while inducing only moderate somnolence (4). Two recent articles confirmed the efficacy and safety of etomidate infusion at these doses in the treatment of severe CS in the intensive care unit (ICU) $(7,8)$. On the other hand, a significant cortisol lowering effect has been demonstrated while using even lower doses of etomidate $(0.03 \mathrm{mg} / \mathrm{kg} / \mathrm{h})$ (4). Based on data obtained in a limited number of patients, Soh et al. (9) also recently argued for the use of etomidate at lower doses in the non-ICU setting. However, it is not yet clear whether very low doses of etomidate can be safely used in the non-critical care setting with an equal efficacy.

In order to further elucidate this question, we studied the outcome of two series of patients with severe and complicated CS treated either with a classical etomidate dose in ICU or with a very low dose in a non ICU setting.

\section{Methods}

We retrospectively analyzed medical records of 14 patients who had been treated with etomidate for a severe CS between 2013 and 2019 in three hospitals in Belgium: Saint Luc University Hospital and Erasme University Hospital in Brussels and Saint Pierre Hospital in Ottignies. Details of each patient (\#1 to 14) are presented in a Supplementary Table 1 (see section on supplementary materials given at the end of this article). In all patients, first-line curative surgery was not possible due to advanced metastatic disease, occurrence of severe complications precluding immediate surgery, or ignorance of the responsible etiology upon admission. In most patients, etomidate infusion was continued until the day of a subsequent surgery, which consisted in unilateral adrenal tumour debulking in five patients (with advanced metastatic ACC), bilateral adrenalectomy in three cases (with EAS), and resection of an ACTH-secreting neoplasm in one patient (Supplementary Table 1). The other patients received chemotherapy $(n=3)$ or palliative care after disease diagnosis and staging $(n=2)$.

Etomidate treatment indications varied, but the rationale was always to obtain a quick control of severe hypercortisolism in order to prevent or correct co-morbidity and to decrease subsequent surgical risk. Etomidate (Hypnomidate ${ }^{\circledR} 20 \mathrm{mg} / 10 \mathrm{~mL}$ in propylene glycol solution, Janssen pharmaceuticals) was administered pure intravenously through a drug pump. Overall, the median duration of etomidate treatment was 10 days (interquartile range IQR: 5-19 days).

We subdivided patients in two groups according to their initial etomidate treatment regimen: either with a very low starting dose in a medical ward (0.020-0.040 $\mathrm{mg} / \mathrm{kg} / \mathrm{h}$ ) or with a high starting dose given in ICU $(0.100-0.300 \mathrm{mg} / \mathrm{kg} / \mathrm{h})$. The type of etomidate regimen and ICU systematic admission or not were mainly based on the local protocols used in the different hospitals: a high dose protocol was performed in Erasme University hospital and a low dose protocol in the two others centers. One patient (patient \# 6, Supplementary Table 1) was first treated for 1 week with a low etomidate dose in ICU because severe CS diagnosis occured during ICU stay for Listeria meningitis treatment. In this case, etomidate treatment was further continued in a medical ward for 8 days before surgery. Therefore, this patient was included in the low dose/medical ward group. Another patient (patient \# 14, Supplementary Table 1) was first treated in ICU with a high starting dose $(0.30 \mathrm{mg} / \mathrm{kg} /$ day $)$ forw 1 day, then etomidate infusion was decreased to low doses $(0.04 \mathrm{mg} / \mathrm{kg} /$ day $)$ in order to maintain cortisol levels in the target range. Nevertheless, this patient was included in the high starting dose/ICU group.

In all but two patients, serum cortisol levels were measured with the Elecsys Cortisol II immunoassay (Roche Diagnostics $\mathrm{GmbH}$ ) which has a low cross-reactivity for 11-deoxycortisol (S), ranging between 3.6 and $4.9 \%$ over time. In two patients treated before June 2014, the Access II Cortisol immunoassay was used (Beckman Coulter Inc., Brea, CA 92821 U.S.A), which suffers from a higher crossreactivity with $\mathrm{S}(17.8 \%)$.

We compared baseline characteristics, treatment response, and subsequent evolution in these two groups. All statistical analyses were performed using the IBM SPPS software version 25.0, using unpaired $t$-tests and chi squared tests for statistical significance with a $P$ value set at 0.05 . Due to their asymmetric distribution, all serum and urinary free cortisol values were log-transformed for statistical comparisons. The study was approved by the Ethics Committee of Saint Luc University Hospital as part of a larger project related to the differential diagnosis and treatment of Cushing's syndrome (reference number 2016/9NOV/486). As this was a retrospective study on medical files, patient's informed consent was not required. All data were handled according to national laws and European general data protection regulation. 


\section{Results}

We identified 14 patients (nine men and five women, mean age \pm S.D.: $56 \pm 18$ years) initially treated with etomidate for severe CS between 2013 and 2019. Median morning serum cortisol level was $1337 \mathrm{nmol} / \mathrm{L}$ (interquartile range (IQR): 1130-1713; normal range: 250-500) and median UFC was $1427 \mu \mathrm{g} / 24 \mathrm{~h}$ (IQR: 757-4535; normal range: 10-60). Mean serum potassium concentration was $2.71 \pm 0.71 \mathrm{mM}$. Thirteen patients (93\%) had hypertension and nine (64\%) had diabetes upon presentation. In all patients, hypercortisolism was secondary to a cancer: eight (57\%) had ACC with ENSAT stage IIIb or IV and six (43\%) had non adrenal neoplasia with EAS leading to secondary CS syndrome. Eleven patients (79\%) had nodal extension of their cancer and ten $(71 \%)$ had distant metastases.

We subdivided patients into two groups according to etomidate dose regimen and the type of unit where treatment was administered. Four patients were treated in the ICU only with a median starting dose of 0.30 $\mathrm{mg} / \mathrm{kg} / \mathrm{h}$ (IQR: 0.15-0.30) ('high dose' regimen). These patients had frequent blood test monitoring, every $6 \mathrm{~h}$ for the duration of treatment. The ten other patients were treated initially in a standard medical ward with a low dose etomidate regimen (except one patient first treated in ICU for 10 days). In this group, the median starting dose was $0.025 \mathrm{mg} / \mathrm{kg} / \mathrm{h}$ (IQR: 0.020-0.031) which was gradually increased by daily increments of $0.010 \mathrm{mg} / \mathrm{kg} / \mathrm{h}$ (or $0.50 \mathrm{mg} / \mathrm{h}$ ) until a morning cortisol level target below $500 \mathrm{nmol} / \mathrm{L}$ was reached. Surveillance was less frequent in the low-dose group: morning potassium and serum cortisol concentrations were measured every $12 \mathrm{~h}$ during the first day and then every $24 \mathrm{~h}$.

Baseline characteristics of the two subgroups are compared in Table 1. There was no significant difference in mean age, tumour type (EAS/ACC), or sex ratio, while the relative proportion of men tended to be higher in the low-dose group $(P=0.052)$. Serum and urinary cortisol levels were not significantly different between groups. Biological disturbances associated with hypercortisolemia (hypokalemia, metabolic alkalosis, and low TSH) were similar. In particular, hypokalemia was mild to moderate in all patients, except in two subjects (one in each subgroup) who had kalemia below $2.0 \mathrm{mmol} / \mathrm{L}$ upon admission (Supplementary Table 1). The mean creatinine level was slightly higher in the lowdose group $(P=0.011)$, perhaps related to gender difference. Interestingly, severe CS complications were similar in both subgroups (Supplementary Table 1) and decision to treat patients at high etomidate dose in ICU setting was not dictated by the need for assisted ventilation or other organsupporting therapy.

The evolution of morning serum cortisol in the two subgroups is reported in Fig. 1 and Table 2. Mean final etomidate dose at steady state remained much lower in the medical ward group (median: $0.030 \mathrm{mg} / \mathrm{kg} / \mathrm{h}$; IQR: $0.020-0.080$ ) as compared with the ICU group (median: $0.100 \mathrm{mg} / \mathrm{kg} / \mathrm{h}$; IQR: $0.040-0.300$ ), although the difference weaned and was no longer significant. Total treatment duration was longer in the low-dose group. Rapid decreases in cortisol levels were observed in both groups. However, as expected, patients in ICU-high-dose group achieved normal cortisol levels faster (median time to reach cortisol levels below $500 \mathrm{nmol} / \mathrm{L}$ : 1 day vs 3 days;

Table 1 Baseline characteristics of patients with severe Cushing syndrome, treated with i.v. infusion of etomidate at a low starting dose in a non-ICU medical ward $(n=10)$ and at a high starting dose in intensive care unit $(I C U)(n=4)$. All values are shown as mean \pm S.D., medians and interquartile ranges, or proportions.

\begin{tabular}{|c|c|c|c|}
\hline & Low-dose etomidate $(n=10)$ & High-dose etomidate $(n=4)$ & $P$ value \\
\hline Starting dose etomidate $(\mathrm{mg} / \mathrm{kg} / \mathrm{h})$ & $0.025(0.020-0.035)$ & $0.300(0.150-0.300)$ & 0.021 \\
\hline Age & $59.3 \pm 14.4$ & $46.8 \pm 26.1$ & NS \\
\hline ICU days & 0 * & $2.0(1.2-5.8)$ & 0.010 \\
\hline Sex ratio M/F & $8 / 2$ & $1 / 3$ & 0.052 \\
\hline Tumour type EAS/ACC & $5 / 5$ & $1 / 3$ & NS \\
\hline Morning serum cortisol (nmol/L) & $1428(1225-1988)$ & $1169(1092-1285)$ & NS \\
\hline 24-h urinary free cortisol ( $\mu \mathrm{g} / 24 \mathrm{~h})$ & $1313(643-7097)$ & $1875(1278-3358)$ & NS \\
\hline Serum potassium (mmol/L) & $2.58 \pm 0.59$ & $3.05 \pm 0.97$ & NS \\
\hline Serum bicarbonate $(\mathrm{mmol} / \mathrm{L})$ & $35.7 \pm 7.6$ & $29.0 \pm 4.8$ & NS \\
\hline Serum creatinine $(\mathrm{mg} / \mathrm{dL})$ & $1.13 \pm 0.48$ & $0.64 \pm 0.07$ & 0.011 \\
\hline Serum TSH (mU/L) & $0.76 \pm 0.79$ & $1.18 \pm 0.68$ & NS \\
\hline
\end{tabular}

All values are shown as mean \pm S.D., medians and interquartile ranges, or proportions. NS, not significant.

*One patient was first treated in ICU during 10 days at low doses of etomidate, then 8 days in the medical ward.

ACC, adrenocortical carcinoma; EAS, ectopic ACTH secretion; ICU, intensive care unit; TSH, thyroid stimulating hormone. 


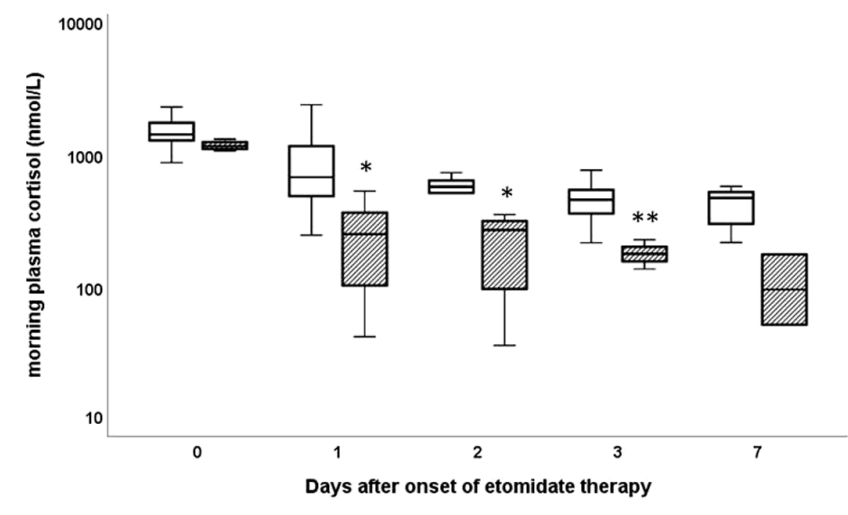

Figure 1

Evolution of morning serum cortisol levels during the first week of etomidate treatment given at low dose in medical ward (white boxes; $n=10$ ) or at high dose in ICU (hatched boxes; $n=4)$. The box-plots represent medians, interquartile range, and P5 and P95 values. * **: $P<0.05$ and 0.01 vs the low-dose group.

$P=0.013$ ), and morning cortisol levels on days 1, 2 and 3 were significantly lower in ICU-high-dose group, while the difference was no longer significant at day 7-10 (Fig. 1). As a consequence of this faster and deeper cortisol decrease, documented hypocortisolism requiring hydrocortisone (HC) supplementation was the rule in ICU-high-dose group, while none of the patients in standard medical ward, low-dose group required HC replacement therapy.

Initial hypokalemia could be rapidly corrected in all patients through combined i.v. and oral administration of potassium $(61 \pm 35 \mathrm{mEq}$ and $65 \pm 38 \mathrm{mEq}$ given on days 1 and 2 after etomidate start, respectively), together with spironolactone $50-200 \mathrm{mg} /$ day in 10 out of 14 patients. Thus, serum potassium concentrations (in $\mathrm{mEq} / \mathrm{L}$ ) rise from $2.8 \pm 0.7$ before etomidate to $3.7 \pm 1.7$ on day 1 and to $4.1 \pm 1.6$ on day 2 , with all patients above 3.0 at that time. There was no difference in potassium evolution between the two dose subgroups.

The main outcome data during and after etomidate treatment are summarized in Table 3. In the medical ward group, etomidate was well tolerated with only one patient experiencing slight somnolence. In contrast, in the ICU group, all patients experienced some degree of sleepiness, but above all required oral cortisol supplementation for adrenal insufficiency. Two deaths were noted during etomidate treatment, both in the medical ward group. These two patients died for oncological reasons after decision was made to enter palliative care: one patient died from a metastatic ACC with massive liver invasion and poor performance status contra-indicating any life-prolonging chemotherapy and the other patient died from respiratory failure secondary to high grade unresectable pulmonary small cell carcinoma. The two groups were comparable in terms of additional treatment, including the use of other cortisol reducing drugs, bilateral adrenalectomy and/or chemotherapy. None of the 14 patients required a second etomidate treatment course. During short-term follow-up, all patients from the ICU group and six patients (60\%) from the medical ward group died, after median intervals of 6.7 (3.5-10.8) and 1.7 (1.3-4.4) months, respectively. The oldest patient in the study (ICU subgroup) died from bilateral pneumonia while hypercorticism was under control. Finally, four patients were still alive during the follow-up period of 10.2 (2.1-32.0) months.

\section{Discussion}

We report for the first time a dose comparison study for patients with severe Cushing syndrome (CS) treated with i.v. etomidate infusion either with a low starting dose in a normal medical ward or with a high starting dose in

Table 2 Morning serum cortisol response to etomidate therapy and need for hydrocortisone supplementation according to the low or high starting dose regimen. All values are shown as medians and interquartile ranges or proportions.

\begin{tabular}{lcc}
\hline & Low-dose etomidate $(n=10)$ \\
\hline Steady state infusion rate $(\mathrm{mg} / \mathrm{kg} / \mathrm{h})$ & & $0.037(0.023-0.059)$ \\
Serum cortisol day $1(\mathrm{nmol} / \mathrm{L})$ & $681(488-1292)$ \\
Serum cortisol day 2 $(\mathrm{nmol} / \mathrm{L})$ & $560(443-690)$ \\
Serum cortisol day $3(\mathrm{nmol} / \mathrm{L})$ & $425(294-576)$ \\
Serum cortisol day $7-10(\mathrm{nmol} / \mathrm{L})$ & $3(2-6)$ \\
Nb of days until cortisol <500 $\mathrm{nmol} / \mathrm{L}$ & $13(9-20)$ \\
Duration of etomidate therapy (days) & $0 / 10$ \\
Hydrocortisone supplementation &
\end{tabular}

\begin{tabular}{c}
\hline High-dose etomidate $(n=4)$ \\
\hline $0.125(0.085-0.263)^{\star}$ \\
$253(95-465)$ \\
$272(93-338))$ \\
$180(138-180)$ \\
$115(52-225)$ \\
$1(1-2)$ \\
$3(2-5)$ \\
$4 / 4$
\end{tabular}

\begin{tabular}{c}
\hline P value \\
\hline NS \\
0.012 \\
0.018 \\
0.005 \\
NS \\
0.013 \\
0.015 \\
0.001
\end{tabular}

values for cortisol levels were calculated after log transformation.

*One patient in the high-dose group was first treated in ICU with a high starting dose $(0.30 \mathrm{mg} / \mathrm{kg} / \mathrm{day}) \mathrm{for} 1 \mathrm{day}$, then the dose was reduced to $0.04 \mathrm{mg} /$ $\mathrm{kg} /$ day in order to maintain cortisol levels in the target range. 
Table 3 Outcome data during and after etomidate treatment for a severe Cushing syndrome, in patients treated with low doses in the medical ward $(n=10)$ or with high doses in the ICU $(n=4)$.

\begin{tabular}{lcc}
\hline & Low-dose etomidate $(n=10)$ \\
\hline Death during etomidate treatment & $2 / 10(20 \%)$ \\
Use of other anticortisol drugs & $4 / 10(40 \%)$ \\
Bilateral adrenalectomy & $2 / 10(20 \%)$ \\
Chemotherapy after etomidate & $5 / 10(50 \%)$ \\
Number of deaths during complete follow-up & $6 / 10(60 \%)$ \\
Duration of follow-up before death (months) & $1.7(1.3-4.4)$ \\
\hline
\end{tabular}

\begin{tabular}{c}
\hline High-dose etomidate $(n=4)$ \\
\hline $0 / 5(0 \%)$ \\
$2 / 4(50 \%)$ \\
$1 / 4(25 \%)$ \\
$2 / 4(50 \%)$ \\
$4 / 4(100 \%)$ \\
$6.7(3.5-10.8)$
\end{tabular}

\begin{tabular}{l} 
P value \\
NS \\
NS \\
NS \\
NS \\
NS \\
NS \\
\hline
\end{tabular}

NS, not statistically significant

an intensive care unit (ICU). Of note, patients in both groups had diseases of comparable severity in terms of serum cortisol concentrations, UFC excretion, potassium and bicarbonate levels, or CS acute complications. The only observed differences were a male predominance and higher creatinine levels in the medical ward group. It is unlikely that these differences may have affected response to etomidate and final outcome, especially as treatment protocol and setting applied mainly depended on local practice and expertise.

These findings also highlight differences in practice and the need for risk stratification strategies for admission to intensive care units. In the United Kingdom, criteria for admission in ICU are defined by the need for advanced respiratory support, support for two or more organ systems, support for one organ system in patients with severe comorbidities or an expected benefit from a more detailed monitoring than could be provided on a general ward (10). In the setting of a severe Cushing syndrome, decision may vary among centers, depending on patient type recruitment, as well as local expertise and facilities.

In case of severe CS, marked hypokalemia could be considered an important reason for admission in the ICU, because of the risk of cardiac arrhythmia. However, in our study, hypokalemia was not less severe in the low-dose group. Moreover, despite an expected etomidate-related accumulation of mineralocorticoids (such as deoxycorticosterone), hypokalemia could be easily corrected in all patients and none of them had complications due to severe hypokalemia. This can be explained by the fact that hypokalemia was mild to moderate $(2.5-3.0 \mathrm{mmol} / \mathrm{L})$ in the majority of our patients and thus, according to recent guidelines (11), could even be treated orally as no symptoms or cardiac rhythm perturbations were present,. Furthermore, a recent study has shown no benefit in terms of cardiac arrhythmia from potassium supplementation in hospitalized patients with mild to moderate hypokalemia (12).

In all our patients with severe CS, etomidate was effective in reducing cortisol levels to the target range (below $500 \mathrm{nmol} / \mathrm{L}$ ) in a few days, whatever the regimen used. However, the high-dose group exhibited a significantly faster and more profound response, cortisol levels being lower and for a longer duration after starting etomidate than in the low-dose group. This rapid and strong effect had the disadvantages to require closer ICU surveillance, to induce cortisol insufficiency and to need hydrocortisone supplementation in all patients treated with high dose etomidate. The low-dose group achieved target cortisol levels slower (3 days) but without adrenal insufficiency and with less medical resources.

To our knowledge, this is only the second report on the use of etomidate in several patients outside an ICU setting. Soh et al. (9) previously reported that the drug could be used safely, without the risk for unwanted sedation, and effectively at a dose of $2-4 \mathrm{mg} / \mathrm{h}$ in hypercortisolic patients who did not require ventilation or inotropic support. The speed of cortisol normalization was, however, not specifically studied in their report. Here, we confirm these data in a significant number of additional patients, using similar low doses of etomidate and a less stringent 24-h monitoring, sufficient to show the time course of cortisol decrease.

In addition to these data, the successful use of a lowdose etomidate protocol has been described recently in an ICU setting. Thus, Carroll et al. reported on seven patients with severe CS treated in ICU with a starting dose of $0.02 \mathrm{mg} / \mathrm{kg} / \mathrm{h}$ of etomidate, which was titrated every $6 \mathrm{~h}$ in order to achieve a target cortisol concentration of 275 to $551 \mathrm{nmol} / \mathrm{L}$ in 24 to $48 \mathrm{~h}$ without the need for cortisol supplementation (8). Patients were given an optional initial $5 \mathrm{mg}$ i.v. bolus of etomidate over 2 to $3 \mathrm{~min}$. This protocol has the advantage of using a low starting dose and small incremental dose adjustments in order to achieve cortisol control without inducing adrenal failure, but requires frequent monitoring and intensive care beds and resources. Despite this, two patients experienced nausea and vomiting close to the end of the infusion that was attributed to the some degree of hypocortisolism. 
In our study, the use of etomidate was safe and well tolerated in both subgroups. This was particularly true in the medical ward group, where only one patient reported mild somnolence. These results are reassuring and should encourage clinicians to consider a wider etomidate use in medical wards for patients in whom intensive care maneuvers or surveillance are not needed. Noticeably, no patient suffered from an acute adrenal crisis or from myoclonus or other side effects possibly related to polyethylene glycol (13).

The final outcome of our patients was poor, mainly due to the severity of the underlying disease causing severe CS. Two patients treated in non-intensive wards died during etomidate treatment, both from their endstage cancer disease and not from consequences of their hypercortisolism. Regarding longer term survival, all patients initially treated in the ICU died, as well as more than half of the patients treated in a medical ward. Clearly, due to the small number of patients and differences in tumour types and stages between the two groups, no conclusion can be drawn regarding the superiority of anyone of the two etomidate regimens for overall survival. It seems, however, fair to say that using lower dose does not adversely affect the long-term prognosis of these patients.

Of note, in patients treated by etomidate (as well as metyrapone), 11-deoxycortisol (S) may be largely increased due to the marked inhibition of 11- $\beta$-hydroxylase. This may lead to an overestimation of true cortisol concentrations, as all currently available cortisol immunoassays express some degree of cross-reactivity with $\mathrm{S}$ due to the similarity of the two molecules. In most patients, however, we used an assay with relatively low cross-reactivity (less than 5\%), so that analytical errors were minimized. Ideally, mass spectrometry should have been be used to measure serum cortisol, as it will prevent this type of interaction (14).

Our study has several limitations. The retrospective nature of our work and the lack of complete data sets for all patients reduced the depth of analysis. Reflecting real practices, no strict protocol was followed and respected in either group. Also, because of the limited number of patients and heterogeneity of underlying diagnoses, no conclusion can be drawn regarding a potential longterm advantage of a very rapid normalization of cortisol levels in ICU over a slower (and possibly) safer decrease in medical ward. However, despite the small patient number, it is still significant because of the rarity of the condition. Our study is the largest one describing the use of etomidate outside ICU. It is also one of the only studies to show that, because of inherent etomidate pharmacological properties, less frequent monitoring is allowed if doses are very low and dose adjustments are incremental. Due to the rarity and potentially lethal complications of severe CS, we strongly advise, however, that these patients be managed in tertiary care centers with a high caseload and experience in the full management of this condition, including availability of a multidisciplinary team for the care of the underlying cause.

In conclusion, i.v. etomidate administration was effective in rapidly reducing cortisol levels to safe levels in all patients with cancer-related severe Cushing's syndrome. The high-dose-ICU group benefited from a faster cortisol response, which, however, did not improve the final outcome. The low-dose-medical-ward group achieved target cortisol levels slower, but without inducing adrenal insufficiency or other side-effects, and without need for intensive care resources. We suggest, therefore, to more often consider the possibility to use very low doses of etomidate in normal medical wards for patients with a severe CS who do not require ventilation or other organ-supporting therapy.

\section{Supplementary materials}

This is linked to the online version of the paper at https://doi.org/10.1530/ EJE-20-0380.

Declaration of interest

The authors declare that there is no conflict of interest that could be perceived as prejudicing the impartiality of this study.

\section{Funding}

This research did not receive any specific grant from any funding agency in the public, commercial or not-for-profit sector.

\section{References}

1 Alexandraki KI \& Grossman AB. Therapeutic strategies for the treatment of severe Cushing's syndrome. Drugs 201676 447-458. (https://doi.org/10.1007/s40265-016-0539-6)

2 Young J, Haissaguerre M, Viera-Pinto O, Chabre O, Baudin E \& Tabarin A. Management of Endocrine Disease: Cushing's syndrome due to ectopic ACTH secretion: an expert operational opinion. European Journal of Endocrinology 2020182 R29-R58. (https://doi. org/10.1530/EJE-19-0877)

3 Baudin E \& Endocrine Tumor Board of Gustave Roussy. Adrenocortical carcinoma. Endocrinology and Metabolism Clinics of North America 201544 411-434. (https://doi.org/10.1016/j. ecl.2015.03.001)

4 Schulte HM, Benker G, Reinwein D, Sippell WG \& Allolio B. Infusion of low dose etomidate: correction of hypercortisolemia in patients with Cushing's syndrome and dose-response relationship in normal 
subjects. Journal of Clinical Endocrinology and Metabolism 199070 1426-1430. (https://doi.org/10.1210/jcem-70-5-1426)

5 Broersen LHA, Jha M, Biermasz NR, Pereira AM \& Dekkers OM. Effectiveness of medical treatment for Cushing's syndrome: a systematic review and meta-analysis. Pituitary 201821 631-641. (https://doi.org/10.1007/s11102-018-0897-z)

6 Daniel E, Aylwin S, Mustafa O, Ball S, Munir A, Boelaert K, Chortis V, Cuthbertson DJ, Daousi C, Rajeev SP et al. Effectiveness of metyrapone in treating Cushing's syndrome: a retrospective multicenter study in 195 patients. Journal of Clinical Endocrinology and Metabolism 2015100 4146-4154. (https://doi.org/10.1210/jc.20152616)

7 Preda VA, Sen J, Karavitaki N \& Grossman AB. Etomidate in the management of hypercortisolaemia in Cushing's syndrome: a review. European Journal of Endocrinology 2012167 137-143. (https://doi. org/10.1530/EJE-12-0274)

8 Carroll TB, Peppard WJ, Herrmann DJ, Javorsky BR, Wang TS, Patel H, Zarnecki K \& Findling JW. Continuous etomidate infusion for the management of severe Cushing syndrome: validation of a standard protocol. Journal of the Endocrine Society 20193 1-12. (https://doi.org/10.1210/js.2018-00269)

9 Soh LM, Gunganah K, Akker SA, Jones P, Khachi H, Dodzo K $\&$ Drake WM. Etomidate in the emergency management of hypercortisolemia. European Journal of Endocrinology 2012167 727-728; author reply 729. (https://doi.org/10.1530/EJE-12-0698)

10 Smith G \& Nielsen M. ABC of intensive care. Criteria for admission. BMJ 1999318 1544-1547. (https://doi.org/10.1136/ bmj.318.7197.1544)

11 Kardalas E, Paschou SA, Anagnostis P, Muscogiuri G, Siasos G \& Vryonidou A. Hypokalemia: a clinical update. Endocrine Connections 20187 R135-R146. (https://doi.org/10.1530/EC-18-0109)

12 Harkness W, Watts P, Kopstein M, Dziadkowiec O, Hicks G \& Scherbak D. Correcting hypokalemia in hospitalized patients does not decrease risk of cardiac arrhythmias. Advances in Medicine 2019 2019 4919707. (https://doi.org/10.1155/2019/4919707)

13 Doenicke A, Roizen MF, Nebauer AE, Kugler A, Hoernecke R $\&$ Beger-Hintzen H. A comparison of two formulations for etomidate, 2-hydroxypropyl- $\beta$-cyclodextrin (HPCD) and propylene glycol. Anesthesia and Analgesia 199479 933-939. (https://doi. org/10.1213/00000539-199411000-00020)

14 Monaghan PJ, Owen LJ, Trainer PJ, Brabant G, Keevil BG \& Darby D. Comparison of serum cortisol measurement by immunoassay and liquid chromatography-tandem mass spectrometry in patients receiving the $11 \beta$-hydroxylase inhibitor metyrapone. Annals of Clinical Biochemistry 201148 441-446. (https://doi.org/10.1258/ acb.2011.011014)

Received 16 April 2020

Revised version received 15 May 2020

Accepted 20 May 2020 